\title{
Wie sieht die optimale Behandlung aus?
}

Bis heute besteht kein Konsens über die optimale Behandlung bei Rupturen des vorderen Kreuzbandes (VKB) bei Kindern und Jugendlichen. Ziel dieser Meta-Analyse war es, die Vorteile und Nachteile von operativer und konservativer Therapie zu analysieren. Ramski et al. verglichen die Ergebnisse aus 11 Studien. Ramski DE, Kanj WW, Franklin CC, Baldwin KD, Ganley T]. Anterior Cruciate Ligament Tears in Children and Adolescents: A Meta-analysis of Nonoperative Versus Operative Treatment. Am J Sports Med. 2013 Dec 4. [Epub ahead of print]

\section{Material und Methoden}

In einer Metaanalyse wurden die Studien nach den folgenden Hauptkriterien eingeschlossen: (1) Englische Sprache (2) Evidenzgrad 1 bis 3 (3) Retrospektives oder prospektives Studiendesign mit Untersuchung konservativer vs. operativer Therapie (4) Retrospektives oder prospektives Studiendesign mit Untersuchung der initialen vs. verzögerten VKB Rekonstruktion. Der Literaturauswahlprozess beinhaltete die Extraktion von Daten basierend auf den folgenden Variablen: symptomatische Meniskusrisse, Rückkehr zu Aktivitäten, klinische Ergebnisse sowie erneute Operation und persistierende Instabili- tät/ pathologische Laxizität. Ein symptomatischer Meniskusriss wurde wie folgt definiert: Auftreten nach der initialen Verletzung, die Begrenzung der Aktivität und die Erfordernis weiterer Behandlung. Eine Instabilität/pathologische Laxizität wurde wie folgt definiert: Ein Nachgeben Grad $\geq 2$ im Lachman/Pivot-Shift- Testergebnis oder ein Unterschied zur kontralateralen gesunden Seite von $>4 \mathrm{~mm}$ in der KT-1000 Messung.

\section{Ergebnisse \\ $\nabla$}

6 Studien (217 Patienten) vergleichen die Rekonstruktion des VKB mit einem kon-

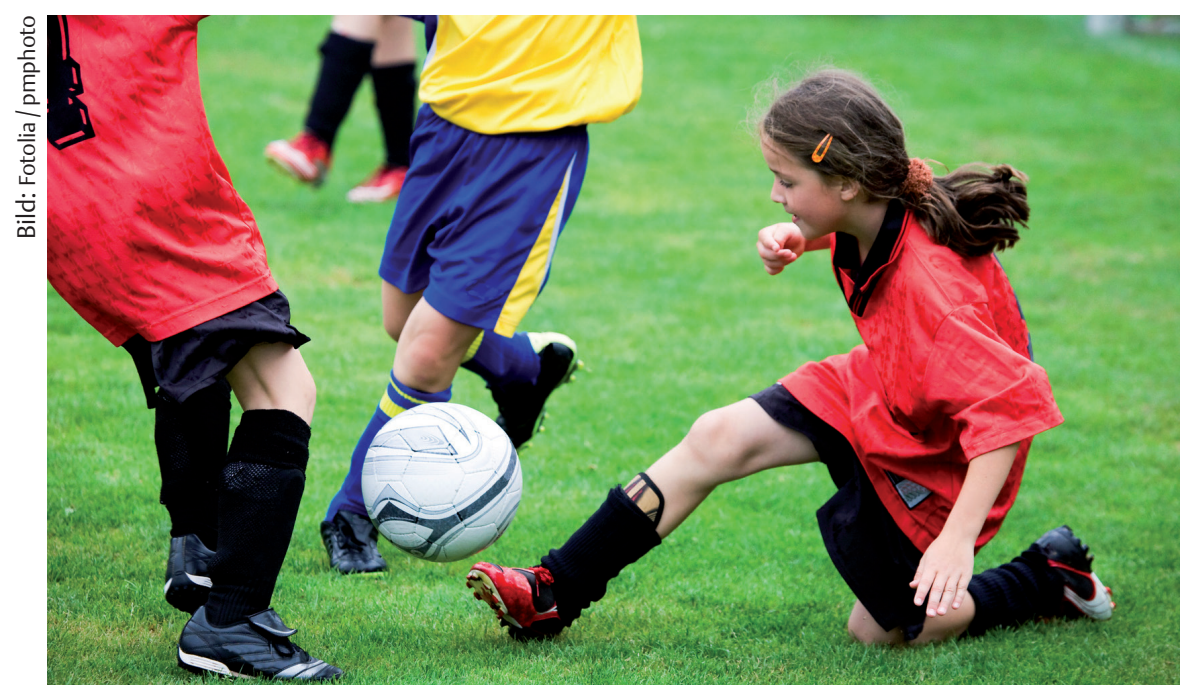

servativen Therapieregime. Weitere5 Studien (353 Patienten) vergleichen eine frühe VKB Rekonstruktion mit einer verzögerten. Konservativ behandelte Patienten zeigten eine $12 x$ höhere Inzidenz von Meniskusrissen im Vergleich zu den operierten Patienten $(35,4 \%$ vs 3,9\%, $\mathrm{P}=0,02)$. Eine Instabilität/pathologische Laxizität fand sich bei 13,6\% der Patienten nach operativer vs. $75 \%$ nach konservativer Therapie $(P<0,01)$. Ein signifikanter Unterschied zwischen den Gruppen im IKDC-Score (International Knee Documentation Committee) wurde in einer von zwei Studien zu Gunsten der operativen Therapie festgestellt $(P=0,002)$. In einer von zwei Studien wurde zudem ein signifikanter Unterschied zu Gunsten des operativen Vorgehens im Tegner-Score festgestellt $(P=0,007)$. Zwei Studien berichteten bezüglich der Rückkehr zur Aktivität, dass keiner der Patienten in den konservativ behandelten Gruppen sein vorheriges Aktivitätsniveau wiedererlangen konnte, im Vergleich zu 85,7\% der Patienten in den operativen Gruppen $(\mathrm{P}<0,01)$.

\section{Kommentar}

$\nabla$

Die Ergebnisse dieser Analyse deuten darauf hin, dass die operierten Patienten ihr vorheriges Aktivitätsniveau zu einem hohen Prozentsatz wiedererlangen, dass sie signifikant weniger Meniskusschäden erleiden und, dass signifikant weniger Instabilitäten zurückbleiben. Die Autoren nehmen in dieser Studie nur Bezug auf die o.g. Parameter. Mögliche Auswirkungen auf die Wachstumsfuge werden nicht berichtet. Weitere aus der Literatur bekannte Daten deuten allerdings darauf hin, dass bei adäquater Technik der Fixierung des Transplantates keine wesentlichen Auswirkungen $\mathrm{zu}$ erwarten sind. Enstprechend sollte man jungen aktiven Sportlern zu einer operativen Versorgung raten.

\section{Dr. Max Ettinger}

Orthopädische Klinik der Medizinischen Hochschule Hannover

Max@ettinger.info 